\title{
ОБ ОПРЕДЕЛЯЕМОСТИ ПЕРИОДИЧЕСКОЙ АБЕЛЕВОЙ ГРУППЫ СВОЕЙ ПОЛУГРУППОЙ ЭНДОМОРФИЗМОВ В КЛАССЕ ВСЕХ ПЕРИОДИЧЕСКИХ АБЕЛЕВЫХ ГРУПП
}

\author{
(Представил А. Хумал)
}

\section{1. Введение}

Известно, что изоморфизм колец эндоморфизмов двух периодических абелевых групп влечет за собой изоморфизм самих этих групп ([ $\left.{ }^{1}\right]$ c. 265). При доказательстве этого утверждения пользуются, как правило, действием сложения эндоморфизмов. Целью настоящей работы является доказательство того, что уже из изоморфизма полугрупп всех эндоморфизмов двух периодических абелевых групп следует изоморфизм самих этих групп.

Введем следующие обозначения: $C_{t}$ - циклическая группа порядка $t ; C\left(p^{\infty}\right)$ - группа типа $p^{\infty}$; End $G$ - полугруппа всех эндоморфизмов группы $G$; Aut $G$ - группа всех автоморфизмов группы $G$; $I(G)$ - совокупность всех идемпотентов полугруппы End $G$; $\langle a\rangle$ - подгруппа, порожденная элементом $a ; o(a)$ - порядок элемента $a$ некоторой группы; $K_{G}(x)=\{y \in$ End $G \mid y x=x y=y\} ; \quad Q_{G}(x, y)=$ $=\{z \in$ End $G \mid x z=z y=z\}$.

Так как множество $K_{G}(x)$ образует полугруппу, то будем рассматривать его как полугруппу. Элеменгы $x$ и $y$ полугруппы End $G$ будем называть ортогональными, если $x y=y x=0$. Для абелевых групп будем пользоваться аддитивной записью.

\section{2. Вспомогательные леммы}

Л ем м а 1. Пусть $G$ - некоторая группа $u x, y \in I(G)$. Тогда множество $Q_{G}(x, y)$ состоит из всевозможных эндоморфизмов z группы $G$, для которых $z: \operatorname{Ker} x \rightarrow\langle 1\rangle u z: \operatorname{Im} x \rightarrow \operatorname{Im} y$. Наоборот, каждый гомоморфизм $z: \operatorname{Im} x \rightarrow \operatorname{Im} y$ однозначно распространим на всю группу $G$ так, что $z: \operatorname{Ker} x \rightarrow\langle 1\rangle$.

Доказ а тельство. Пусть $x, y \in I(G)$ и предположим, что $z \in$ $\in Q_{G}(x, y)$. Тогда $x z=z y=z$. Поэтому $($ Ker $x) z=\langle 1\rangle$. Так как

$$
\operatorname{Im} y=\{g \in G \mid g y=g\}
$$

(см. $\left[{ }^{2}\right]$, лемма 1.1), то ввиду $z y=z$ получаем включение $(\operatorname{Im} x) z \subset$ $\subset \operatorname{Im} y$. Пусть, наоборот, $z \in \operatorname{End} G,(\operatorname{Ker} x) z=\langle 1\rangle$ и $(\operatorname{Im} x) z \subset \operatorname{Im} y$. Поскольку $G=\operatorname{Ker} x \lambda \operatorname{Im} x\left({ }^{2}\right]$, лемма 1.1$)$, то $G z=(\operatorname{Im} x) z \subset \operatorname{Im} y$, откуда в силу равенства (1) имеем $z y=z$. Так как идемпотент $x$ действует на $\operatorname{Im} x=G x$ тождественно и $(\operatorname{Ker} x) x=(\operatorname{Ker} x) z=\langle 1\rangle$, то 
$x z=z$. Следовательно, $z \in Q_{G}(x, y)$ и первое утверждение леммы доказано. Второе утверждение леммы следует сразу, ибо имеет место разложение $G=\operatorname{Ker} x \lambda \operatorname{Im} x$. Лемма доказана.

Согласно доказанному, можем отождествить всякий эндоморфизм $z \in Q_{G}(x, y)$ соответствующим гомоморфизмом $z: \operatorname{Im} x \rightarrow \operatorname{Im} y$. Ясно, что при $x, y, u \in I(G)$ всегда $Q_{G}(x, y) Q_{G}(y, u) \subset Q_{G}(x, u)$. Так как $Q_{G}(x, x)=K_{G}(x)$, то из леммы 1 сразу следует

Л е м м а 2. Eсли $x \in I(G)$, то $K_{G}(x) \simeq \operatorname{End}(\operatorname{Im} x)$.

Лемм а 3. Пусть абелева группа $A$ разлагается на прямую сумму $A=\langle a\rangle \oplus\langle b\rangle \oplus C$, где $a, b-p$-элементы, для которых $о(b) \leqslant o(a), u$ $\varepsilon_{a}, \varepsilon_{b}$ - проекции группь $A$ на подгруппь $\langle a\rangle u\langle b\rangle$ соответственно. Если $\xi \in Q_{A}\left(\varepsilon_{a}, \varepsilon_{b}\right), \quad a \xi=b$, то существует единственный $\pi \in$ $\in Q_{A}\left(\varepsilon_{a}, \varepsilon_{a}+\varepsilon_{b}\right)$, так что $\pi \varepsilon_{a}=\varepsilon_{a} u \pi \varepsilon_{b}=\xi$. При этом $\pi=\varepsilon_{a}+\xi$.

Док а з а тельство. Пусть выполнены предположения леммы. Определим гомоморфизм $\pi: \operatorname{Im} \varepsilon_{a}=\langle a\rangle \rightarrow \operatorname{Im}\left(\varepsilon_{a}+\varepsilon_{b}\right)=\langle a\rangle \oplus\langle b\rangle$ равенством $a \pi=a+b$. В силу $o(b) \leqslant o(a)$ отображение $\pi$ действительно определяет гомоморфизм, а также эндоморфизм из $Q_{A}\left(\varepsilon_{a}, \varepsilon_{a}+\varepsilon_{b}\right)$, причем $a \pi=a+b=a \varepsilon_{a}+a \xi=a\left(\varepsilon_{a}+\xi\right)$, т. е. $\pi=$ $=\varepsilon_{a}+\xi$. Так как $\xi, \varepsilon_{a}, \pi \varepsilon_{a} \in Q_{A}\left(\varepsilon_{a}, \varepsilon_{a}+\varepsilon_{b}\right)$ и $a\left(\pi \varepsilon_{a}\right)=(a+b) \varepsilon_{a}=$ $=a \varepsilon_{a}, a\left(\pi \varepsilon_{b}\right)=(a+b) \varepsilon_{b}=b=a \xi$, то $\pi \varepsilon_{a}=\varepsilon_{a}$ и $\pi \varepsilon_{b}=\xi$. Если еще $\tau \in Q_{A}\left(\varepsilon_{a}, \varepsilon_{a}+\varepsilon_{b}\right)$ и $\tau \varepsilon_{a}=\varepsilon_{a}, \tau \varepsilon_{b}=\xi$, то $a \tau=s a+t b$ для некоторых целых чисел $s, t$ и

$$
\begin{gathered}
a=a \varepsilon_{a}=a\left(\tau \varepsilon_{a}\right)=(s a+t b) \varepsilon_{a}=s a, \\
b=a \xi=a\left(\tau \varepsilon_{b}\right)=(s a+t b) \varepsilon_{b}=t b,
\end{gathered}
$$

т. е. $s=t=1$ и $a \tau=a+b=a \pi, \tau=\pi$. Лемма доказана.

Л емм а 4. Пусть абелева группа $A$ разлагается на прямую сумму $A=\langle a\rangle \oplus\langle b\rangle \oplus B$ и $\varepsilon_{a}, \varepsilon_{b}$ - проекции группь $A$ на подгруппьь $\langle a\rangle u\langle b\rangle$ соответственно. Если $\alpha \in Q_{A}\left(\varepsilon_{a}, 1\right)$ u $\beta \in Q_{A}\left(\varepsilon_{b}, 1\right)$, то во множестве $Q_{A}\left(\varepsilon_{a}+\varepsilon_{b}, 1\right)$ существует единственный элемент $\xi$, так что $\varepsilon_{a} \xi=\alpha$, $\varepsilon_{b} \xi=\beta$. При этом $\xi=\alpha+\beta$.

Доказ ательство. Пусть выполнены предположения леммы. Определим $\xi \in Q_{A}\left(\varepsilon_{a}+\varepsilon_{b}, 1\right)$ равенством $\xi=\alpha+\beta$. Тогда $\varepsilon_{a} \xi=$ $=\varepsilon_{a}(\alpha+\beta)=\varepsilon_{a} \alpha+\varepsilon_{a} \beta=\alpha+0=\alpha$ и, аналогично, $\varepsilon_{b} \xi=\beta$. Предположим, что $\tau \in Q_{A}\left(\varepsilon_{a}+\varepsilon_{b}, 1\right)$ и $\varepsilon_{a} \tau=\alpha, \quad \varepsilon_{b} \tau=\beta$. Тогда $a \tau=$ $=(a+0) \tau=\left(a \varepsilon_{a}+a \varepsilon_{b}\right) \tau=a\left(\varepsilon_{a} \tau\right)+a\left(\varepsilon_{b} \tau\right)=a \alpha+a \beta=a(\alpha+\beta)$.

Аналогично, $b \tau=b(\alpha+\beta)$. Следовательно, $\tau=\alpha+\beta=\xi$. Лемма доказана.

Л ем м а 5. Если $G$ и Н некоторые группы, $x \in I(G)$, $\operatorname{Im} x-$ конечная абелева групnа $u$ End $G \simeq$ End $H$, то $\operatorname{Im} x \simeq \operatorname{Im} x^{*}$, где $x^{*}$ - образ идемпотента х при изоморфизме End $G \simeq$ End $H$.

Доказательство. Пусть выполнены предположения леммы. Ясно, что $K_{G}(x) \simeq K_{H}\left(x^{*}\right)$. По лемме 2 End $(\operatorname{Im} x) \simeq \operatorname{End}\left(\operatorname{Im} x^{*}\right)$. Тогда $\operatorname{Im} x \simeq \operatorname{Im} x^{*}$, ибо каждая конечная абелева группа определяется своей полугруппой эндоморфизмов в классе всех групп (см. $\left[{ }^{2}\right]$, теорема 4.2). Лемма доказана.

Предположим, что группа $A$ абелева и $x \in I(A)$. Тогда $A=$ $=\operatorname{Ker} x \lambda \operatorname{Im} x=\operatorname{Ker} x \oplus \operatorname{Im} x$ (см. $\left[{ }^{2}\right]$, лемма 1.1) и $x$ является проекцией группы $A$ на ее подгруппу $\operatorname{Im} x$. В дальнейшем будем через $x^{T}$ обозначать проекцию группы $A$ на подгруппу $\operatorname{Ker} x$. Назовем идемпотент $x^{T}$ ортогональным дополнением к идемпотенту $x$. Ясно, что 
$\left(x^{T}\right)^{T}=x$. Для $x \in I(A)$ его ортогональное дополнение определено однозначно равенствами $x x^{T}=x^{T} x=0$ и $x+x^{T}=x^{T}+x=1$.

Л ем м а 6. Пусть группа $A$ абелева и идемпотенты $x_{1}, x_{2}, \ldots, x_{n} \in$ $\in I(A)$ nопарно-ортогональны. Если End $A \simeq$ End $B$, где $B$ - некоторая другая абелева группа, то

$$
\left(x_{1}+x_{2}+\ldots+x_{n}\right)^{*}=x_{1}^{*}+x_{2}^{*}+\ldots+x_{n}^{*},
$$

где $x^{*}$ - образ элемента $x \in$ End $A$ при изоморфизме End $A \simeq$ End $B$.

Доказательство этой леммы непосредственно вытекает из следствий $1.9,1.11$ и 1.12 работы $\left[{ }^{2}\right]$. При $n=2$ и $x_{2}=x_{1}{ }^{T}$ из леммы 6 следует, что $\left(x_{1}^{T}\right)^{*}=\left(x_{1}{ }^{*}\right)^{T}$.

Л ем м а 7. Если End $C\left(p^{\infty}\right) \simeq$ End $C\left(q^{\infty}\right)$, то $p=q$.

Доказ ательство. В силу $\left[{ }^{1}\right]$ (с. 372 ) группа Aut $C\left(p^{\infty}\right)$ изоморфна группе $C_{p-1} \oplus J_{p}$, если $p>2$, и группе $C_{2} \oplus J_{2}$, если $p=2$ $\left(J_{p}\right.$ - аддитивная группа кольца целых $p$-адических чисел). Группа $J_{p}$ является группой без кручения. Поэтому из изоморфизма End $C\left(p^{\infty}\right) \simeq$ End $C\left(q^{\infty}\right)$ следует изоморфизм $J_{p} \simeq J_{q}$. Тогда изоморфны также кольца эндоморфизмов групп $J_{p}$ и $J_{q}$, т. е. кольца целых $p$-адических и $q$-адических чисел (см. $\left[{ }^{1}\right]$, с. 256$)$. Последний изоморфизм возможен лишь при $p=q$. Лемма доказана.

Л ем ма 8. Если группа $A$ абелева $и$ End $A \simeq \operatorname{End} C\left(p^{\infty}\right)$, то $A \simeq$ $\simeq C\left(p^{\infty}\right)$.

Доказа тельство. Пусть группа $A$ абелева и End $A \simeq$ End $C\left(p^{\infty}\right)$. Так как полугруппа End $C\left(p^{\infty}\right)$ изоморфна мультипликативной полугруппе кольца целых $p$-адических чисел ([ $\left.{ }^{1}\right]$, с. 256), то полугруппа End $A$ бесконечна и лишена делителей нуля. В $\left.{ }^{3}{ }^{3}\right]$ доказано, что такая группа $A$ изоморфна группе $C_{q}$ или группе $C\left(q^{\infty}\right)$ для некоторого простого числа $q$. Случай $A \simeq C_{q}$ исключается, ибо полугруппа End $A$ бесконечна. Поэтому $A \simeq C\left(q^{\infty}\right)$ и End $A \simeq$ End $C\left(p^{\infty}\right) \simeq$ End $C\left(q^{\infty}\right)$. В силу леммы $7 p=q$. Следовательно, $A \simeq C\left(p^{\infty}\right)$. Лемма доказана.

\section{3. Основная теорема}

Основным результатом нашей работы является следующая

Т еорема. Если полугруппь всех эндоморфизмов двух периодических абелевых групп $A$ и $B$ изоморфны, то группы $A$ и $B$ изоморфны.

Для доказательства теоремы нам потребуется еще ряд лемм.

Л ем м а 9. Если $A$ - делимая периодическая абелева группа, $B$ периодическая абелева группа $и$ End $A \simeq \operatorname{End} B$, то $A \simeq B$.

Доказ ательство. Пусть выполнены предположения леммы. Обозначим через $x^{*}$ образ элемента $x \in$ End $A$ при изоморфизме End $A \simeq$ $\simeq$ End $B$. Ввиду делимости группы $A$ имеем $A=\oplus_{i \in J} A_{i}, \quad A_{i} \simeq$ $\simeq C\left(p_{i}{ }^{\infty}\right) \quad$ (см. $\left[{ }^{4}\right]$, с. 124$)$. Обозначим через $\varepsilon_{i}$ проекцию группы $A$ на прямое слагаемое $A_{i}$. Ясно, что множество $T=\left\{\varepsilon_{i} \mid i \in J\right\}$ является системой попарно-ортогональных идемпотентов полугруппы End $A$, причем имеет место

У слов и е. $Е с л и \varepsilon \in I(A)$ и $\varepsilon \varepsilon_{i} \varepsilon=0$ для каждого $i \in J$, то $\varepsilon=0$. Тогда $T^{*}=\left\{\varepsilon_{i}{ }^{*} \mid i \in J\right\}$ является системой попарно-ортогональных идемпотентов полугруппы End $B$ и $K_{A}\left(\varepsilon_{i}\right) \simeq K_{B}\left(\varepsilon_{i}{ }^{*}\right)$. 
Обозначим $B_{i}=\operatorname{Im} \varepsilon_{i}{ }^{*}$. Согласно лемме 5 имеем $B_{i}=\operatorname{Im} \varepsilon_{i}{ }^{*} \simeq$ $\simeq \operatorname{Im} \varepsilon_{i} \simeq C\left(p_{i}^{\infty}\right)$. В силу ортогональности системы $T^{*}$ ясно, что

$$
B_{i} \cap\left(B_{1}+\ldots+B_{n}\right)=\langle 0\rangle
$$

при различных $i, 1, \ldots, n$ из множества индексов $J$. Поэтому подгруппы $B_{i}, i \in J$, порождают прямую сумму $C=\bigoplus_{i \in J} B_{i} \simeq \bigoplus_{i \in J} C\left(p_{i}^{\infty}\right)$ в группе $B$. Группа $C$ как делимая подгруппа выделяется в виде прямого слагаемого из группы $B$ :

$$
B=C \oplus D=\left(\oplus_{i \in J} B_{i}\right) \oplus D .
$$

Обозначим через $\varepsilon^{*}$ проекцию группы $B$ на подгруппу $D$. Тогда $\varepsilon^{*} \in$ $\in I(B), \quad \bigoplus_{i \in J} B_{i} \subset$ Ker $\varepsilon^{*} \quad$ и $\varepsilon_{i}{ }^{*} \varepsilon^{*}=0^{*}$ для каждого $i \in J$. Поэтому $\varepsilon \in I(A)$ и $\varepsilon_{i} \varepsilon=0$ для каждого $i \in J$. Согласно условию имеем $\varepsilon=0$. Следовательно, $\varepsilon^{*}=0^{*}, D=\langle 0\rangle$ и $B=C=\oplus_{i \in J} B_{i} \simeq \oplus_{i \in J} A_{i}=A, \quad$ ибо $B_{i} \simeq A_{i} \simeq C\left(p_{i}^{\infty}\right)$ для каждого $i \in J$. Лемма доказана.

Если $A$ - периодическая абелева группа, то через $A_{p}$ будем обозначать примарную $p$-компоненту группы $A$.

Л ем м а 10. Если $A$ - редуцированная периодическая абелева группа, $A=\oplus_{p \in P} A_{p} \quad\left(A_{p} \neq\langle 0\rangle\right.$ при $\left.p \in P\right), B-$ периодическая абелева apynna $u$ End $A \simeq$ End $B$, то $B=\oplus_{p \in P} B_{p} \quad u$ End $A_{p} \simeq$ End $B_{p} \partial \Omega r$ каждого $p \in P$.

Доказательств о. Пусть выполнены предположения леммы. Обозначим через $x^{*}$ образ элемента $x \in \operatorname{End} A$ при изоморфизме End $A \simeq$ $\simeq$ End $B$ и через $\varepsilon_{p}$ проекцию группы $A$ на подгруппу $A_{p}$. В силу реду. цированности группы $A$ группа $A_{p}$ обладает нетривиальным циклическим прямым слагаемым $\left\langle a_{p}\right\rangle$ (см. [ $\left.{ }^{4}\right]$, с. 140, следствие 27.3):

$$
A_{p}=\left\langle a_{p}\right\rangle \oplus \bar{A}_{p}, \quad p \in P .
$$

Тогда

$$
A=\left\langle a_{p}\right\rangle \oplus \bar{A}_{p} \oplus\left(\oplus_{q \in P \backslash\{p\}} A_{q}\right) .
$$

Обозначим через $\delta_{p}$ проекцию группы $A$ на подгруппу $\left\langle a_{p}\right\rangle$ и рассмотрим множество

$$
T_{p}=\left\{x \in I(A) \mid Q_{A}\left(\delta_{p}, x\right)=\{0\}\right\} .
$$

Предположим, что $x \in T_{p}$. Тогда $Q_{A}\left(\delta_{p}, x\right)=\{0\}$. Согласно определению множества $Q_{A}\left(\delta_{p}, x\right)$ это означает, что всякий гомоморфизм $\operatorname{Im} \delta_{p}=\left\langle a_{p}\right\rangle \rightarrow \operatorname{Im} x$ является нулевым. Отсюда следует, что группа $\operatorname{Im} x$ лишена неединичных $p$-элементов. Следовательно,

$$
T_{p}=\left\{x \in I(A) \mid \operatorname{Im} x-p^{\prime} \text {-группа }\right\} .
$$

Поэтому и $\varepsilon_{p}{ }^{T} \in T_{p}$.

Установим, что идемпотент $\varepsilon_{p}{ }^{T}$ является двусторонней единицей множества $T_{p}$. Пусть $x \in T_{p}$. В силу равенства (2) $\operatorname{Im} x \subset \operatorname{Ker} \varepsilon_{p}$ и $\operatorname{Im} \varepsilon_{p} \subset \operatorname{Ker} x$, т. е. $\varepsilon_{p} x=x \varepsilon_{p}=0$. Поэтому

$$
x=x 1=x\left(\varepsilon_{p}+\varepsilon_{p}^{T}\right)=x \varepsilon_{p}+x \varepsilon_{p}^{T}=0+x \varepsilon_{p}^{T}=x \varepsilon_{p}^{T} .
$$

Аналопично $x=\varepsilon_{p}{ }^{T} x$. Следовательно, $\varepsilon_{p}{ }^{T}-$ двусторонняя единица множества $T_{p}$.

Рассмотрим теперь группу $B$. Предположим, что $B=\otimes_{q \in Q} B_{q}$, и

2 ENSV TA Toimetised F* M-3 1980 
обозначим через $\pi_{q}$ проекцию группы $B$ на ее подгруппу $B_{q}$. Так как $\operatorname{Im} \delta_{p}=\left\langle a_{p}\right\rangle$ - ненулевая циклическая $p$-группа $(p \in P)$ и по лемме $5 \mathrm{Im} \delta_{p}{ }^{*} \simeq\left\langle a_{p}\right\rangle$, то $P \subset Q$.

Покажем, что End $A_{p} \simeq$ End $B_{p}$ для каждого $p \in P \subset Q$. Пусть $p \in P$. По определению множества $Q(\cdot, \cdot)$ ясно, что $\left(Q_{A}\left(\delta_{p}, x\right)\right)^{*}=$ $=Q_{B}\left(\delta_{p}^{*}, x^{*}\right)$. Поэтому

$$
T_{p}^{*}=\left\{x^{*} \in I(B) \mid Q_{B}\left(\delta_{p}^{*}, x^{*}\right)=\left\{0^{*}\right\}\right\},
$$

причем $\left(\varepsilon_{p}^{T}\right)^{*}$ - двусторонняя единица множества $T_{p}^{*}$, ибо $\varepsilon_{p}^{T}-$ двусторонняя единица множества $T_{p}$. Аналогично тому, как это делалось при $T_{p}$, можно убедиться, что

$$
T_{p}^{*}=\left\{x^{*} \in I(B) \mid \operatorname{Im} x^{*}-p^{\prime} \text {-группа }\right\}
$$

и $\pi_{p}{ }^{T}$ является двусторонней единицей множества $T_{p}{ }^{*}$. Так как двусторонняя единица определена однозначно, то $\left(\varepsilon_{p}^{T}\right)^{*}=\pi_{p}^{T}$. Поэтому $\pi_{p}=\left(\pi_{p}^{T}\right)^{T}=\left(\left(\varepsilon_{p}^{T}\right)^{*}\right)^{T}=\left(\left(\varepsilon_{p}^{T}\right)^{T}\right)^{*}=\varepsilon_{p}{ }^{*}$. В силу изоморфизма $K_{A}\left(\varepsilon_{p}\right) \simeq K_{B}\left(\varepsilon_{p}^{*}\right)=K_{B}\left(\pi_{p}\right)$ и леммы 2 End $A_{p} \simeq$ End $B_{p}$.

Установим, наконец, равенство $P=Q$. По равенству (2) ясно, что

$$
\bigcap_{p \in P} T_{p}=\left\{x \in I(A) \mid \operatorname{Im} x-P^{\prime} \text {-группа }\right\} .
$$

Согласно равенству $A=\oplus_{p \in P} A_{p}$ это означает, что $\bigcap_{p \in P} T_{p}=\{0\}$. Поэтому и $\bigcap_{p \in P} T_{p}^{*}=\left\{0^{*}\right\}$. В силу равенства (3) имеем

$$
\bigcap_{p \in P} T_{p}^{*}=\left\{x^{*} \in I(B) \mid \operatorname{Im} x^{*}-P^{\prime} \text {-группа }\right\}=\left\{0^{*}\right\} .
$$

Если $P \neq Q$, то существует $q \in Q \backslash P$. Тогда $\pi_{q} \neq 0^{*}, \pi_{q} \in I(B)$ и $\operatorname{Im} \pi_{q}$ является ненулевой $P^{\prime}$-группой. Это противоречит равенству (4). Следовательно, $P=Q$. Лемма доказана.

Лемм а 11. Если $A$ - неограниченная редуцированная периодическая абелева р-группа, то группа $A$ имеет неограниченную базисную. nодарупny.

Док аз ательство. Группа $A$ имеет базисную подгруппу $B$, причем $B=\oplus_{n=1}^{\infty} B_{n}$, где $B_{n}$ - прямая сумма групп $C_{p^{n}}$ или $B_{n}=\langle 1\rangle$ (см. $\left[{ }^{4}\right]$, c. $\left.162-164\right)$. Предположим от противного, что группа $B$ ограничена. Тогда существует число $k>0$ такое, что $B_{k}=B_{k+1}=\ldots=\langle 0\rangle$, $B=B_{1} \oplus \ldots \oplus B_{k-1}$ и $A=B \oplus C$ для некоторой подгруппы $C$. Группа $C$ лишена ненулевого циклического прямого слагаемого, ибо группа $B_{1} \oplus \ldots \oplus B_{n}$ является максимальным $p^{n}$-ограниченным прямым слагаемым группы $A$ (см. $\left[{ }^{4}\right]$, с. 166 , теорема 33.2$)$. Отсюда следует, что $C=\langle 0\rangle$, так как при $C \neq\langle 0\rangle$ в силу редуцированности группы $C$ следовало бы существование ненулевого циклического прямого слагаемого для группы $C$ (см. $\left[{ }^{4}\right]$, с. 140$)$. Поэтому $A=B=$ $=B_{1} \oplus \ldots \oplus B_{k-1}$, т. е. группа $A$ ограничена. Это противоречит предположениям леммы. Следовательно, базисная подгруппа $B$ группы $A$ тоже неограничена. Лемма доказана.

Л ем м а 12. Если $A$ - неограниченная редуцированная периодическая абелева p-группа, $B-$ периодическая абелева р-группа $u$ End $A \simeq$ $\simeq$ End $B$, то группы $A$ и В изоморфны.

Доказ ательство. Пусть выполнены предположения леммы. По лемме 11 группа $A$ имеет неограниченную базисную подгруппу. Поэтому существуют элементы $a_{1}, a_{2}, \ldots$ группы $A$ такие, что 


$$
\begin{aligned}
& A=\left\langle a_{1}\right\rangle \oplus \ldots \oplus\left\langle a_{k}\right\rangle \oplus A_{k} ; \\
& A_{k}=\left\langle a_{k+1}\right\rangle \oplus A_{k+1}, \quad k=1,2, \ldots ; \\
& o\left(a_{k}\right)=p^{n_{k}}, \quad 1 \leqslant n_{1}<n_{2}<\ldots .
\end{aligned}
$$

(см. $\left[{ }^{4}\right]$, с. 163$)$. Обозначим через $\varepsilon_{k}$ проекцию группы $A$ на ее подгруппу $\left\langle a_{k}\right\rangle$ относительно указанных прямых разложений. Тогда $\operatorname{Im} \varepsilon_{k}=\left\langle a_{k}\right\rangle$ и $\left\{\varepsilon_{1}, \varepsilon_{2}, \ldots\right\}$ - бесконечная система попарно-ортогональных идемпотентов. Система $\left\{\varepsilon_{1}{ }^{*}, \varepsilon_{2}{ }^{*}, \ldots\right\}$ ( $x^{*}-$ образ элемента $x \in$ $\in$ End $A$ при изоморфизме End $A \simeq$ End $B$ ) состоит также из попарноортогональных идемпотентов. По лемме $6\left(\varepsilon_{1}+\ldots+\varepsilon_{k}\right)^{*}=\varepsilon_{1}{ }^{*}+\ldots$ $\ldots+\varepsilon_{k}{ }^{*}$. Поэтому индукцией по $k$ легко убедиться, что в группе $B$ имеют место аналогичные разложения:

$$
\begin{aligned}
& B=\operatorname{Im} \varepsilon_{1}^{*} \oplus \ldots \oplus \operatorname{Im} \varepsilon_{k}^{*} \oplus B_{k}, \\
& B_{k}=\operatorname{Im} \varepsilon_{k}^{*} \oplus B_{k+1}, \quad k=1,2,3, \ldots,
\end{aligned}
$$

причем $\varepsilon_{k}{ }^{*}$ - проекция группы $B$ на $\operatorname{Im} \varepsilon_{k}{ }^{*}$ относительно таких прямых разложений. По лемме $5 \operatorname{Im} \varepsilon_{k}{ }^{*} \simeq \operatorname{Im} \varepsilon_{k}=\left\langle a_{k}\right\rangle$. Поэтому существуют $c_{k} \in B, k=1,2, \ldots$, такие, что $\operatorname{Im} \varepsilon_{k}^{*}=\left\langle c_{k}\right\rangle \simeq\left\langle a_{k}\right\rangle$.

Определим для каждых $i, j \in\{1,2, \ldots\}, i \geqslant j$, эндоморфизмы $\xi_{i j} \in Q_{A}\left(\varepsilon_{i}, \varepsilon_{j}\right)$ следующим образом: $a_{i} \xi_{i j}=a_{j}$. Тогда эндоморфизмы $\xi_{i j}(i \geqslant j)$ характеризуются свойствами:

1) из $\xi_{i j} \xi=0, \xi \in K_{A}\left(\varepsilon_{j}\right)$ следует $\xi=0$;

2) $\xi_{i j} \xi_{j k}=\xi_{i k}(i \geqslant j \geqslant k)$;

3) $\xi_{i i}=\varepsilon_{i}$

(первое свойство следует из равенства $K_{A}\left(\varepsilon_{j}\right)=Q_{A}\left(\varepsilon_{j}, \varepsilon_{j}\right)$ и описания множества $\left.Q_{A}(\cdot, \cdot)\right)$. В силу изоморфизма End $A \simeq$ End $B$ эндоморфизмы $\xi_{i j}{ }^{*}$ группы $B$ принадлежат множеству $Q_{B}\left(\varepsilon_{i}{ }^{*}, \varepsilon_{j}{ }^{*}\right)$ и обладают аналогичными свойствами:

$\left.1^{*}\right)$ из $\xi_{i j}{ }^{*} \xi^{*}=0^{*}, \xi^{*} \in K_{B}\left(\varepsilon_{j}^{*}\right)$ следует $\xi^{*}=0 *$;

$\left.2^{*}\right) \xi_{i j}{ }^{*} \xi_{j k}{ }^{*}=\xi_{i k}{ }^{*}(i \geqslant j \geqslant k)$;

$\left.3^{*}\right) \xi_{i i}{ }^{*}=\varepsilon_{i}{ }^{*}$.

Из включения $\xi_{i j}{ }^{*} \in Q_{B}\left(\varepsilon_{i}{ }^{*}, \varepsilon_{j}{ }^{*}\right)$ следует, что $c_{i} \xi_{i j}{ }^{*} \in\left\langle c_{j}\right\rangle(i \geqslant j)$. Свойство $\left.1^{*}\right)$ означает, что $\left\langle c_{i} \xi_{i j}^{*}\right\rangle=\left\langle c_{j}\right\rangle$. Индукцией по $k$ легко убедиться, что элементы $c_{1}, c_{2}, \ldots$ можно выбрать так, чтобы $c_{k+1} \xi_{k+1, k}=c_{k}$. В силу свойств $\left.2^{*}\right)$ и $\left.3^{*}\right)$ тогда $c_{i} \xi_{i j}{ }^{*}=c_{j}(i \geqslant j)$.

Определим теперь отображение $\varphi: A \rightarrow B$, которое является изоморфизмом прупп $A$ и $B$. Пусть $a \in A$. Тогда существует $\alpha \in Q_{A}\left(\varepsilon_{k}, 1\right)$ такой, что $a=a_{k} \alpha$ для некоторого $k$. Действительно, в силv условий $(5)$ и периодичности группы $A$ существует $k$ такой, что $o\left(a_{k}\right) \geqslant o(a)$. Эндоморфизм $\alpha \in Q_{A}\left(\varepsilon_{k}, 1\right)$ можно выбрать так: $a_{k} \alpha=a$, $\left(\right.$ Ker $\left.\varepsilon_{k}\right) \alpha=$ $=\langle 0\rangle$.

Определим далее отображение $\varphi: A \rightarrow B$ с равенством

$$
a \varphi=\left(a_{k} \alpha\right) \varphi=c_{k} \alpha^{*} .
$$

Покажем, что $\varphi$ определено корректно, т. е. если к тому же $\beta \in$ $\in Q_{A}\left(\varepsilon_{j}, 1\right)$ и $a=a_{j} \beta$, то $c_{k} \alpha^{*}=c_{j} \beta^{*}$. Ради определенности предположим, что $j \geqslant k$. Тогда $a_{j} \xi_{j k}=a_{k}, a_{j} \xi_{j k} \alpha=a_{k} \alpha=a=a_{j} \beta \quad$ и $\quad \xi_{j k} \alpha=\beta$, откуда следует, что $\xi_{j k}{ }^{*} \alpha^{*}=\beta^{*}$ и 


$$
c_{k} \alpha^{*}=\left(c_{j} \xi_{j k}^{*}\right) \alpha^{*}=c_{j}\left(\xi_{j k}^{*} \alpha^{*}\right)=c_{j} \beta^{*} .
$$

Следовательно, отображение $\varphi$ определено корректно. Аналогично можно определить отображение $\psi: B \rightarrow A$. При этом отображения $\varphi$ и $\psi$ взаимно обратны. Поэтому отображение $\varphi$ является биекцией.

Покажем, что $\varphi$ является гомоморфизмом. Пусть $a, b \in A$ и установим, что $(a+b) \varphi=a \varphi+b \varphi$. Существуют $\alpha \in Q_{A}\left(\varepsilon_{k}, 1\right), \quad \beta \in$ $\in Q_{A}\left(\varepsilon_{l}, 1\right)$ такие, что $a=a_{k} \alpha$ и $b=a_{l} \beta$. Можно предполагать, что $k \neq l$ (при $k=l$ имеем $b=a_{k} \beta=a_{k+1}\left(\xi_{k+1, k} \beta\right)$ и вместо $\beta$ можно рассматривать $\left.\xi_{k+1, k} \beta\right)$. Без ограничения общности положим, что $k \geqslant l$. По лемме 3 существует единственное $\pi \in Q_{A}\left(\varepsilon_{k}, \varepsilon_{k}+\varepsilon_{l}\right)$, удовлетворяющее равенствам $\pi \varepsilon_{k}=\varepsilon_{k}$ и $\pi \varepsilon_{l}=\xi_{k l}$, причем $\pi=\varepsilon_{k}+\xi_{k l}$. По лемме 4 существует единственное $\xi \in Q_{A}\left(\varepsilon_{k}+\varepsilon_{l}, 1\right)$, так что $\varepsilon_{k} \xi=\alpha$ и $\varepsilon_{l} \xi=\beta$. При этом $\xi=\alpha+\beta$. Тогда $\pi \xi \in Q_{A}\left(\varepsilon_{k}, 1\right)$ и

$$
\begin{aligned}
a_{k}(\pi \xi) & =\left(a_{k}\left(\varepsilon_{k}+\xi_{k l}\right)\right) \xi=\left(a_{k}+a_{l}\right) \xi= \\
& =a_{k} \xi+a_{l} \xi=a_{k}(\alpha+\beta)+a_{l}(\alpha+\beta)= \\
& =a_{k} \alpha+0+0+a_{l} \beta=a+b .
\end{aligned}
$$

Так как при изоморфизме End $A \simeq$ End $B$ сохраняется сумма ортогональных идемпотентов (см. лемму 6), то $\left(\varepsilon_{k}+\varepsilon_{l}\right)^{*}=\varepsilon_{k}{ }^{*}+\varepsilon_{l}{ }^{*}$. Этот изоморфизм отображает при каждых $x, y \in I(A)$ множество $Q_{A}(x, y)$ на множество $Q_{B}\left(x^{*}, y^{*}\right)$. Поэтому $\pi^{*} \in Q_{B}\left(\varepsilon_{k}{ }^{*}, \varepsilon_{k}{ }^{*}+\varepsilon_{l}{ }^{*}\right)$ и $\xi^{*} \in$ $\in Q_{B}\left(\varepsilon_{k}{ }^{*}+\varepsilon_{l}{ }^{*}, 1^{*}\right)$ являются единственными элементами из этих множеств такими, что $\pi^{*} \varepsilon_{k}{ }^{*}=\varepsilon_{k}{ }^{*}, \pi^{*} \varepsilon_{l}{ }^{*}=\xi_{k l}{ }^{*}, \varepsilon_{k}{ }^{*} \xi^{*}=\alpha^{*}$ и $\varepsilon_{l}{ }^{*} \xi^{*}=\beta^{*}$. По леммам 3 и $4 \pi^{*}=\varepsilon_{k}{ }^{*}+\xi_{k l}{ }^{*}$ и $\xi^{*}=\alpha^{*}+\beta^{*}$. Аналогично равенству (6) получаем равенство

$$
c_{k}\left(\pi^{*} \xi^{*}\right)=c_{k} \alpha^{*}+c_{l} \beta^{*} .
$$

В силу определения отображения $\varphi$ из-равенств (6) и (7) следует, что

$$
\begin{aligned}
(a+b) \varphi & =\left(a_{k}(\pi \xi)\right) \varphi=c_{k}(\pi \xi)^{*}=c_{k}\left(\pi^{*} \xi^{*}\right)= \\
& =c_{k} \alpha^{*}+c_{l} \beta^{*}=\left(a_{k} \alpha\right) \varphi+\left(a_{l} \beta\right) \varphi= \\
& =a \varphi+b \varphi .
\end{aligned}
$$

Следовательно, $\varphi$ является гомоморфизмом. Ранее было установлено, что $\varphi-$ биекция. Поэтому группы $A$ и $B$ изоморфны. Лемма доказана.

Доказательство те оре мы. Пусть $A$ и $B-$ периодические абелевы труппы и End $A \simeq$ End $B$. Группа $A$ разлагается на прямую сумму $A=F \oplus D$, где $D$ - делимая подгруппа и $F-$ редуцированная подгруппа. Тогда группа $B$ разлагается на прямую сумму $B=K \oplus H$, где End $F \simeq$ End $K$ и End $D \simeq$ End $H$ (см. $\left[{ }^{2}\right]$, теорема 1.13). По лемме 9 группы $D$ и $H$ изоморфны. Докажем, что $F \simeq K$. Ввиду леммы 10 для этого достаточно показать, что $F_{p} \simeq K_{p}$. Из этой же леммы следует, что End $F_{p} \simeq$ End $K_{p}$. Если группа $F_{p}$ ограничена, то $F_{p} \simeq K_{p}$ (см. $\left[{ }^{5}\right]$, теорема). Если группа $F_{p}$ неограничена, то по лемме $12 F_{p} \simeq K_{p}$. Следовательно, $F \simeq K$ и $A \simeq B$. Теорема доказана. 


\section{Л ИТ Р Р А Т Р А}

1. Фукс Л., Бесконечные абелевы группы, т. 2, М., «Мир», 1977.

2. П у у сем п П., Уч. зап. Тартуск. ун-та, № 366, 76-104 (1975).

3. S z el e, T., Publ. Math. Debrecen, 1, 89-91 (1949).

4. Ф у к с Л., Бесконечные абелевы группы, т. 1, М., «Мир», 1974.

5. Пу у с е м П П., Изв. АН ЭССР, Физ. Матем., 29, № 3, 241-245 (1980),

Таллинский политехнический институт
Поступила в редакцию 27/III 1980

\section{P. PUUSEMP}

\section{PERIOODILISE ABELI RUHMA MÄARATAVUSEST OMA ENDOMORFISMIPOOL- RUHMAGA PERIOODILISTE ABELI RUHMADE KLASSIS}

On teada, et kahe perioodilise Abeli rühma endomorfismiringide isomiorfismist järeldub nende rühmade eneste isomorfism (vt. [ $\left.{ }^{1}\right]$, lk. 265). Artikli $\left[{ }^{2}\right]$ tulemuste põhjal on võimalik seda omadust üldistada. Käesolevas töös on näidatud, et juba kahe perioodilise Abeli rühma endomorfismipoolrühmade isomorfismist järeldub nende rühmade isomorfism.

\section{P. PUUSEMP}

\section{ON THE DETERMINITY OF A PERIODIC ABELIAN GROUP BY ITS SEMIGROUP OF ENDOMORPHISMS IN THE CLASS OF ALL PERIODIC ABELIAN GROUPS}

All the endomorphisms of an abelian group $A$ form a ring. The abelian group $A$ is characterized by this ring. For example, it is well known that from the isomorphism of rings of all endomorphisms of two periodic abelian groups $A$ and $B$ follows the isomorphism of groups $A$ and $B$ (see $\left[{ }^{1}\right]$, p. 265). All the endomorphisms of an arbitrary group $A$ form only a semigroup End $A$. Let $\mathfrak{A}$ be a class of groups and $A$ a fixed group. If from the isomorphism of semigroups of all endomorphisms of groups $A$ and $B \in \mathscr{U}$ follows the isomorphism of groups $A$ and $B$ then we say that the group $A$ is determined by its semigroup of endomorphisms in the class $\mathfrak{A}$. For arbitrary group $A$ it is interesting to find classes of groups, in which $A$ is determined by its semigroup of endomorphisms. Some results on this problem are presented in the papers $\left[{ }^{2,5}\right]$. For example, in the paper $\left[{ }^{2}\right]$ it is shown, that an arbitrary finite abelian group is determined by its semigroup of endomorphisms in the class of all groups. In the paper $\left[{ }^{5}\right]$ it is proved that an arbitrary abelian group with bounded orders of its elements is determined by its semigroup of endomorphisms in the class of all groups. In this paper, using the methods and results of the papers $\left[{ }^{2,5}\right]$, it is shown that an arbitrary periodic abelian group is determined by its semigroup of endomorphisms in the class of all periodic abelian groups. 